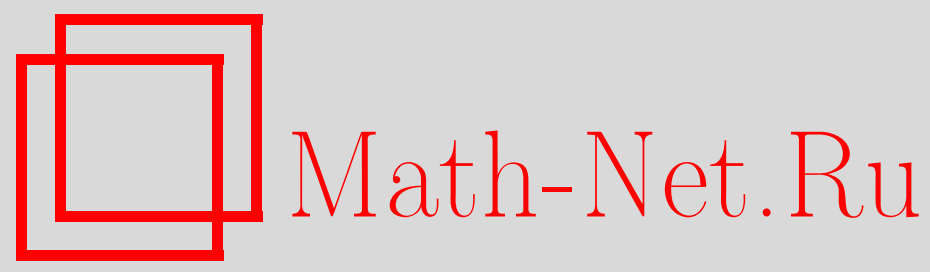

А. С. Бобкова, А. Ю. Колесов, Н. Х. Розов, Проблема "выживания уток" в трехмерных сингулярно возмущенных системах с двумя медленными переменными, Матем. заметки, 2002, том 71, выпуск 6, 818-831

DOI: https://doi.org/10.4213/mzm387

Использование Общероссийского математического портала Math-Net.Ru подразумевает, что вы прочитали и согласны с пользовательским соглашением http://www . mathnet.ru/rus/agreement

Параметры загрузки:

IP : 54.89 .56 .158

26 апреля 2023 г., $12: 28: 51$

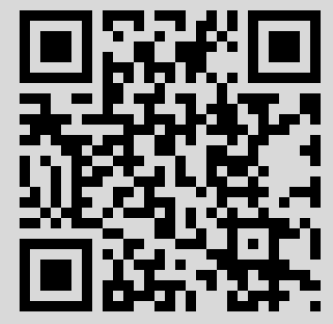




\title{
ПРОБЛЕМА “ВЫЖИВАНИЯ УТОК" В ТРЕХМЕРНЫХ СИНГУЛЯРНО ВОЗМУЩЕННЫХ СИСТЕМАХ С ДВУМЯ МЕДЛЕННЫМИ ПЕРЕМЕННЫМИ
}

\author{
А. С. Бобкова, А. Ю. Колесов, Н. Х. Розов
}

Рассматривается система обыкновенных дифференциальных уравнений $\dot{x}=f(x, y)$, $\varepsilon \dot{y}=g(x, y)$, где $x \in \mathbb{R}^{2}, y \in \mathbb{R}, 0<\varepsilon \ll 1, f, g \in C^{\infty}$. Предполагается, что уравнение $g=0$ определяет две различные гладкие поверхности $y=\varphi(x)$ и $y=\psi(x)$, пересекающиеся общим образом по кривой $l$. Предполагается далее, что траектории соответствующей вырожденной системы, лежащие на поверхности $y=\varphi(x)$, являются утками, т.е. с течением времени, пересекая общим образом кривую $l$, переходят с устойчивой части $\left\{y=\varphi(x), g_{y}^{\prime}<0\right\}$ этой поверхности на неустойчивую ее часть $\left\{y=\varphi(x), g_{y}^{\prime}>0\right\}$. Решается так называемая проблема "выживания уток", т.е. дается ответ на вопрос: какие траектории из имеющегося при $\varepsilon=0$ однопараметрического семейства уток являются пределами при $\varepsilon \rightarrow 0$ некоторых траекторий исходной системы.

Библиограффия: 7 названий.

Впервые траектории-утки были обнаружены в 1978 году французскими математиками $\Phi$. Диенер и М. Диенером с помошью нестандартного анализа. В дальнейшем траектории-утки изучались другими авторами - как методом нестандартного анализа, так и в рамках классического асимптотического анализа. В [1] показано, что при определенных предположениях сингулярно возмущенная система обыкновенных дифференциальных уравнений с малым параметром при части производных может быть представлена как $C^{1}$-возмущение соответствующей релейной системы. Нарушение же этих предположений, как правило, дает начало траекториям-уткам.

В статье [2] рассматривалось скалярное сингулярно возмушенное дифференциальное уравнение первого порядка (что равносильно изучению системы двух дифференциальных уравнений с одной быстрой и одной медленной переменной) и исследовалась проблема возникновения траекторий-уток в случае, когда две различные кривые медленных движений пересекаются обшим образом. В данной работе изучается аналогичньй вопрос, но для системы трех уравнений с одной быстрой и двумя медленньми переменными. При этом без дополнительных пояснений используются понятия и термины, заимствованные из [1], [3].

1. Описание результата. Рассмотрим сингулярно возмушенную систему с одной быстрой и двумя медленными переменными

$$
\dot{x}=f(x, y), \quad \varepsilon \dot{y}=g(x, y),
$$

(C) А. С. БовковА, А. Ю. КолеСов, Н.Х. Розов 
где $x \in \mathbb{R}^{2}, y \in \mathbb{R}, 0<\varepsilon \ll 1$. Предположим, что функции $f$ и $g$ бесконечно дифференцируемы в области $\Omega=\Omega_{x} \times \Omega_{y}$, где $x \in \Omega_{x}, y \in \Omega_{y}$, в которой данная система и будет исследоваться.

УСЛовИЕ 1. Уравнение $g(x, y)=0$ имеет ровно два решения $y=\varphi(x) u y=\psi(x)$, где $\varphi, \psi \in C^{\infty}\left(\Omega_{x}\right)$, а отвечающие им поверхности медленных движений

$$
\Gamma_{1}=\left\{(x, y): x \in \Omega_{x}, y=\varphi(x)\right\}, \quad \Gamma_{2}=\left\{(x, y): x \in \Omega_{x}, y=\psi(x)\right\}
$$

пересекаются общим образом по некоторой гладкой кривой $l$ (последнее означает, что в каждой точке этой кривой выполняется неравенство $\nabla \varphi(x)-\nabla \psi(x) \neq 0)$. Считаем еще, что $g_{y y}^{\prime \prime} \neq 0$ в точках кривой $l$.

Для описания следующего предположения введем в рассмотрение участки

$$
\Gamma_{1}^{-}=\left\{(x, y) \in \Gamma_{1}: g_{y}^{\prime}(x, y)<0\right\}, \quad \Gamma_{1}^{+}=\left\{(x, y) \in \Gamma_{1}: g_{y}^{\prime}(x, y)>0\right\}
$$

поверхности $\Gamma_{1}$, которые будем называть ее устойчивой и неустойчивой частями соответственно.

УСЛОвИЕ 2. При $(x, y) \in \Gamma_{1} \backslash l$ выполняется неравенство $g_{y}^{\prime}(x, y) \neq 0$ и непустым является кахдое из мнохеств (3) (см. рис. 1, на котором изобрахено одно из возможкных расположений поверхностей (2) и участков (3)).
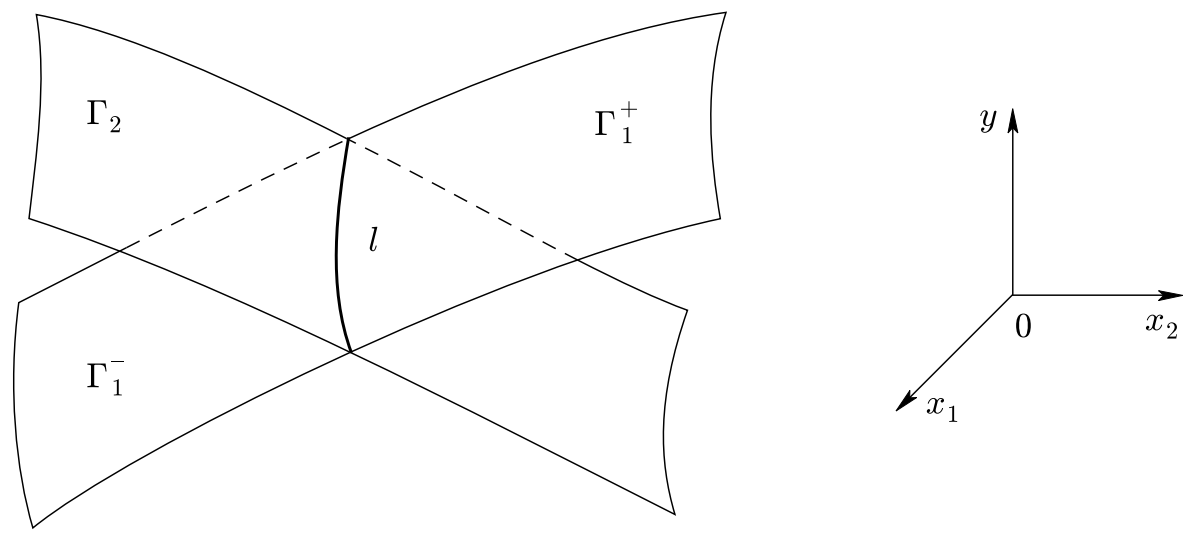

Рис. 1

Обозначим, далее, через $l_{0}$ проекцию кривой $l$ на плоскость $y=0$, а через $\Gamma_{0}^{-}$и $\Gamma_{0}^{+}-$ проекции на плоскость $y=0$ соответственно участков $(3)$ поверхности $\Gamma_{1}$. И наконец, рассмотрим систему

$$
\dot{x}=f(x, \varphi(x))
$$

траектории которой также будем изображать в плоскости $y=0$. 


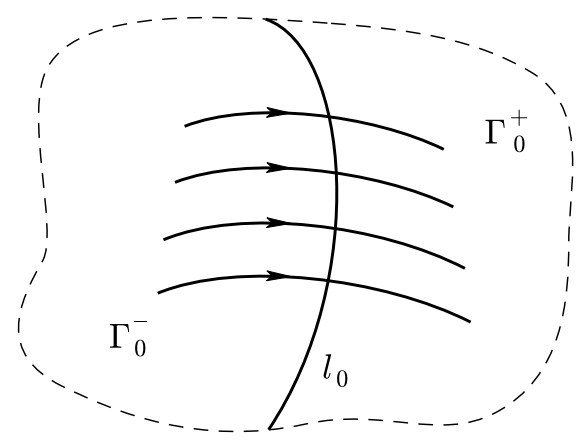

Рис. 2

УСловиЕ 3. Каждая траектория системы (4) пересекает кривую $l_{0}$ общим образом в направлении из $\Gamma_{0}^{-}$в $\Gamma_{0}^{+}$(см. рис. 2).

Для пояснения смысла условия 3 фиксируем произвольно точку $x_{0} \in l_{0}$ и обозначим через $x=x\left(t, x_{0}\right)$ решение системы (4) с начальным условием $\left.x\right|_{t=0}=x_{0}$. Рассмотрим затем отвечающую системе (1) вырожденную систему

$$
\dot{x}=f(x, y), \quad g(x, y)=0
$$

и семейство ее траекторий

$$
(x(t), y(t)): \quad x=x\left(t, x_{0}\right), \quad y=\varphi\left(x\left(t, x_{0}\right)\right), \quad x_{0} \in l_{0},
$$

лежащих на поверхности $\Gamma_{1}$. Согласно терминологии из [1] все эти траектории являются утками, так как при движении по каждой из них фазовая точка системы (5) переходит с устойчивого участка поверхности $\Gamma_{1}$ на неустойчивьй.

Итак, при выполнении условий 1-3 мы сталкиваемся с весьма любопытной ситуацией: при $\varepsilon=0$ у системы (1) имеется однопараметрический континуум траекторий-уток (6). В связи с этим естественньм образом возникает проблема "выживания уток", т.е. вопрос: при каких дополнительных условиях участок $(x(t), y(t)),-t_{1} \leqslant$ $t \leqslant t_{2}$, траектории (6) с наперед заданньми значениями $x_{0} \in l_{0}, t_{j}>0, j=1,2$, является пределом при $\varepsilon \rightarrow 0$ некоторой траектории исходной системы (1)?

Для решения поставленной проблемы фиксируем произвольно точку $x_{0} \in l_{0}$ и отрезок времени $-t_{1} \leqslant t \leqslant t_{2}, t_{j}>0, j=1,2$, а соответствуюший участок траектории $x=x\left(t, x_{0}\right)$ системы (4) обозначим через $x=x_{0}(t)$. (Числа $t_{j}, j=1,2$, выбираются конечными, т.е. не зависящими от $\varepsilon$, но не слишком большими - чтобы указанный участок траектории целиком лежал в допустимой окрестности кривой $l_{0}$.) Зададим, далее, на кривой $l_{0}$ гладкую параметризацию $l_{0}=\{x=\gamma(s), a \leqslant s \leqslant b\}$ и введем в рассмотрение функцию

$$
\Phi(s)=\left.(\nabla \varphi(x), f(x, \varphi(x)))\right|_{x=\gamma(s)} .
$$

Пусть выбранному нами значению $x_{0} \in l_{0}$ соответствует значение параметра $s_{0} \in(a, b)$, т.е. $x_{0}=\gamma\left(s_{0}\right)$. 


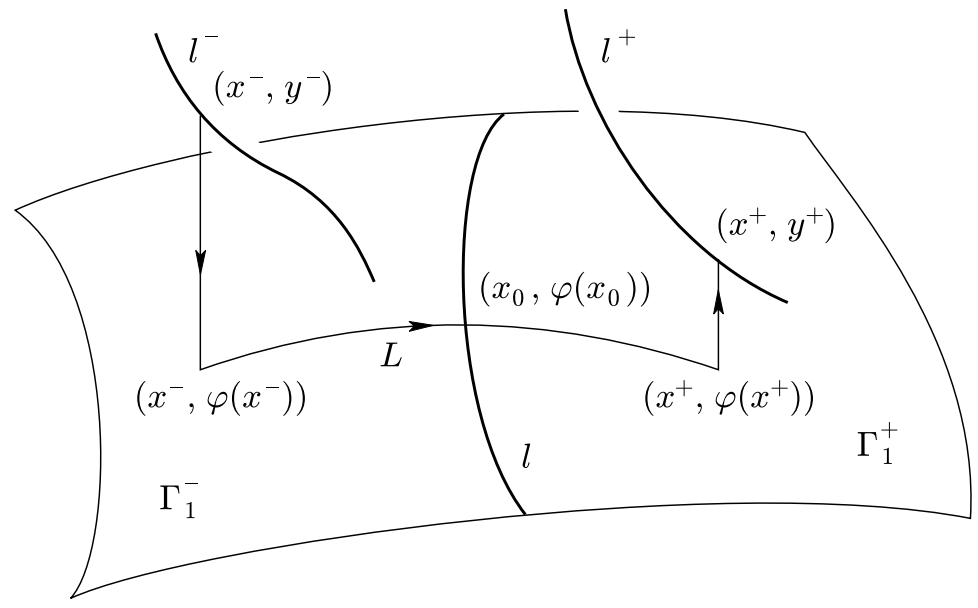

Рис. 3

УСловие 4. Считаем, что $\Phi\left(s_{0}\right)=0, \Phi^{\prime}\left(s_{0}\right) \neq 0$.

Сформулированные условия позволяют дать ответ на интересующий нас вопрос. Однако получение соответствующего строгого результата требует ряда дополнительных построений.

Для их описания положим $x^{-}=x_{0}\left(-t_{1}\right), x^{+}=x_{0}\left(t_{2}\right)$. Рассмотрим, далее, в трехмерном пространстве $\left(x_{1}, x_{2}, y\right)$ две такие кривые $l^{-}$и $l^{+}$, проекции $l_{0}^{+}$и $l_{0}^{-}$которых на плоскость $y=0$ пересекают общим образом траекторию $x=x_{0}(t)$ в точках $x^{-}$и $x^{+}$ соответственно (см. рис. 3,4$)$. И наконец, определим $y^{-}$и $y^{+}$из условий $\left(x^{-}, y^{-}\right) \in l^{-}$ и $\left(x^{+}, y^{+}\right) \in l^{+}$.

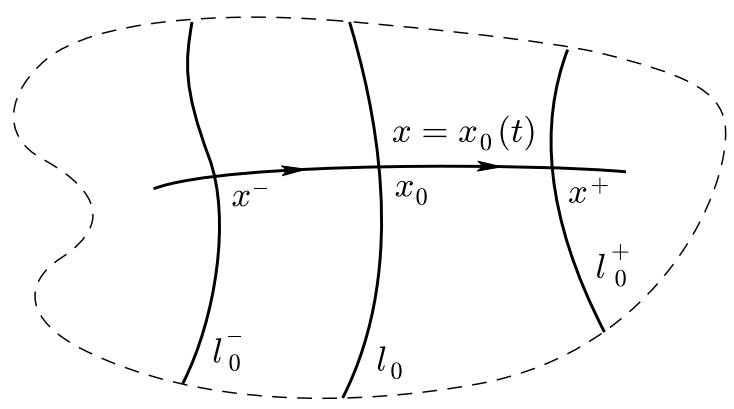

Рис. 4

Пусть $y^{-}(\tau)$ и $y^{+}(\tau)-$ решения задач в “быстром" времени $\tau=t / \varepsilon$ :

$$
\frac{d y}{d \tau}=g\left(x^{-}, y\right), \quad y(0)=y^{-}, \quad \frac{d y}{d \tau}=g\left(x^{+}, y\right), \quad y(0)=y^{+} .
$$

Будем считать, что

$$
y^{-}(\tau) \rightarrow \varphi\left(x^{-}\right) \text {при } \tau \rightarrow+\infty ; \quad y^{+}(\tau) \rightarrow \varphi\left(x^{+}\right) \text {при } \tau \rightarrow-\infty .
$$


Отметим, что вьполнения предельных равенств (9) всегда можно добиться за счет произвола в выборе кривых $l^{-}$и $l^{+}$. Действительно, в силу условий 1,2 фигурирующие в (8) уравнения имеют состояния равновесия $y=\varphi\left(x^{-}\right)$и $y=\varphi\left(x^{+}\right)$соответственно, первое из которых экспоненциально устойчиво в сторону возрастания $\tau$, а второе при $\tau \rightarrow-\infty$. Поэтому равенства (9) будут заведомо справедливы, если взять $y^{-}$и $y^{+}$ достаточно близкими к $\varphi\left(x^{-}\right)$и $\varphi\left(x^{+}\right)$соответственно.

На заключительном этапе рассмотрим кривую $L$, состоящую из двух параллельных оси $y$ отрезков, соединяющих точки $\left(x^{-}, y^{-}\right)$и $\left(x^{-}, \varphi\left(x^{-}\right)\right),\left(x^{+}, y^{+}\right)$и $\left(x^{+}, \varphi\left(x^{+}\right)\right)$, и из лежащего на $\Gamma_{1}$ участка кривой $\left(x_{0}(t), \varphi\left(x_{0}(t)\right)\right)$ при $-t_{1} \leqslant t \leqslant t_{2}$ (см. рис. 3$)$.

ТЕОРемА. При выполнении условий 1-4 существует единственная траектория $L(\varepsilon)$ системы (1) такая, что ее концы лежстн на $l^{-}$u $l^{+}$соответственно, а ее пределом при $\varepsilon \rightarrow 0$ служит кривая $L$.

Траектория $L(\varepsilon)$ системы (1), доставляемая теоремой, и является искомой, так как при $\varepsilon \rightarrow 0$ она неограниченно приближается как к участку кривой $L$, лежащему между точками $\left(x^{-}, \varphi\left(x^{-}\right)\right)$и $\left(x_{0}, \varphi\left(x_{0}\right)\right)$ на устойчивой части $\Gamma_{1}^{-}$, так и к участку кривой $L$, лежашему между точками $\left(x_{0}, \varphi\left(x_{0}\right)\right)$ и $\left(x^{+}, \varphi\left(x^{+}\right)\right)$на неустойчивой части $\Gamma_{1}^{+}$. Обладающую такими свойствами траекторию принято назьвать траекторией-уткой системы (1).

2. Доказательство теоремы. Основная идея обоснования сформулированного результата состоит в сведении задачи к локальной. Для того чтобы сделать это, при $t>0$ рассмотрим пучок траекторий системы $(1)$, начальные условия $\left(x_{*}, y_{*}\right)$ которых при $t=0$ лежат на кривой $l^{-}$в некоторой достаточно малой окрестности $U$ точки $\left(x^{-}, y^{-}\right)$. Опишем поведение каждой из этих траекторий при увеличении $t$.

Согласно обшим результатам монографии [4] сначала (на отрезке вида $0 \leqslant t \leqslant N \varepsilon \times$ $\ln (1 / \varepsilon)$, где $N=$ const $>0)$ происходит так назьваемое быстрое движение в асимптотически малой окрестности прямой $x=x_{*}$, описываемое задачей Коши $(\tau=t / \varepsilon)$

$$
\frac{d y}{d \tau}=g\left(x_{*}, y\right), \quad y(0)=y_{*}
$$

Обратим внимание, что в силу первого предельного равенства $(9)$ и близости $\left(x_{*}, y_{*}\right) \mathrm{k}$ $\left(x^{-}, y^{-}\right)$решение $y(\tau)$ задачи (10) близко к решению первой из задач Коши (8), а значит, оно определено при всех $\tau \geqslant 0$ и удовлетворяет предельному равенству

$$
\lim _{\tau \rightarrow+\infty} y(\tau)=\varphi\left(x_{*}\right)
$$

Таким образом, происходит “падение” каждой из рассматриваемых траекторий на устойчивое многообразие $\Gamma_{1}^{-}$, а затем движение продолжается в его окрестности порядка $\varepsilon$ примерно по закону $(x(t), y(t)), t \geqslant 0$, где $y(t)=\varphi(x(t))$, а $x(t)$ - решение задачи Коши

$$
\dot{x}=f(x, \varphi(x)),\left.\quad x\right|_{t=0}=x_{*} .
$$

Добавим, что в силу условия 3 и близости $x_{*}$ к $x^{-}$решение задачи $(11)$ с течением времени пересекает общим образом кривую $l_{0}$ в некоторой точке, близкой к $x_{0}$. 
Суммируя все сказанное, отметим, что рассмотренный пучок траекторий системы (1) образует гладкое по совокупности переменных интегральное многообразие медленных движений (ленту) $y=S_{1}(x, \varepsilon)$, где $\varepsilon>0, S_{1}(x, 0)=\varphi(x)$, и, что самое главное, данная лента продолжается до пересечения с любой не зависящей от $\varepsilon$ окрестностью точки $\left(x_{0}, \varphi\left(x_{0}\right)\right)$, а точнее говоря - до пересечения с частью этой окрестности, проецируемой на $\Gamma_{1}^{-}$. Аналогичным образом, рассматривая малую окрестность $V \subset l^{+}$ точки $\left(x^{+}, y^{+}\right)$и выпуская при $t<0$ траектории системы (1) из точек множества $V$, получаем ленту $y=S_{2}(x, \varepsilon)$, где $\varepsilon>0, S_{2}(x, 0)=\varphi(x)$, которая также может быть продолжена до пересечения с упомянутой выше окрестностью точки $\left(x_{0}, \varphi\left(x_{0}\right)\right)$, а именно с той ее частью, которая проецируется на $\Gamma_{1}^{+}$. Тем самьм, интересующая нас проблема сводится к локальному вопросу о нахождении в некоторой малой окрестности указанной точки траектории системы $(1)$, концы которой лежат на лентах $y=S_{1}(x, \varepsilon)$ и $y=S_{2}(x, \varepsilon)$.

Следующий этап доказательства - приведение исходной системы (1) в окрестности точки $\left(x_{0}, \varphi\left(x_{0}\right)\right)$ к так назьваемой нормальной форме. Ее построение разбивается, в свою очередь, на несколько шагов. На первом шаге обратимся к условиям 1 и 2 , из которых вытекает равенство

$$
g(x, y)=\omega(x, y)(y-\varphi(x))(y-\psi(x))
$$

где множитель $\omega(x, y) \in C^{\infty}(\Omega)$ отличен от нуля во всех точках поверхности $\Gamma_{1}$. Поэтому без ограничения общности будем считать, что вьполняется неравенство $\omega(x, y)>0$, соответствующее показанному на рис. 1 расположению поверхностей (2) и участков (3) (случай $\omega(x, y)<0$ сводится к данному посредством замены $y$ на $-y$ ). Выполняя затем в системе (1) последовательно замены

$$
\tau=\int_{0}^{t} \omega(x(s, \varepsilon), y(s, \varepsilon)) d s, \quad \tau \rightarrow t, \quad \frac{f(x, y)}{\omega(x, y)} \rightarrow f(x, y), \quad z=y-\varphi(x)
$$

преобразуем ее к виду

$$
\begin{aligned}
\dot{x} & =f(x, z+\varphi(x)), \\
\varepsilon \dot{z} & =(\varphi(x)-\psi(x)) z+z^{2}-\varepsilon(\nabla \varphi(x), f(x, z+\varphi(x))) .
\end{aligned}
$$

Для описания очередной замены переменных фиксируем произвольно точку $x \in \Omega_{x}$ и выпустим из нее траекторию системы (4), т.е. рассмотрим решение $w(t, x)$ этой системы с начальным условием $w(0, x)=x$. Согласно условию 3 существует единственньй момент времени $t_{0}=t_{0}(x)$ (положительный при $x \in \Gamma_{0}^{-}$и отрищательньй при $x \in \Gamma_{0}^{+}$), для которого $w\left(t_{0}, x\right) \in l_{0}$. Обозначим, далее, через $s=s(x)$ значение параметра $s$, отвечающее точке $w\left(t_{0}(x), x\right)$, и перейдем в системе $(4)$ к новым координатам $(\xi, v)$ :

$$
s(x)-s_{0}=v, \quad t_{0}(x)=\xi,
$$

где, напомним, $s_{0}$ - значение параметра $s$, соответствующее выбранной нами точке $x_{0} \in$ $l_{0}$ (см. рис. 4). В итоге рассматриваемая система преобразуется к виду

$$
\dot{\xi}=1, \quad \dot{v}=0
$$


а интересуюшее нас ее решение $x=x_{0}(t)$ в новых переменных задается равенствами

$$
\xi=t, \quad v=0
$$

На втором шаге построения нормальной формы выполним в системе (12) замену (13). В результате с учетом равенств (14) она примет вид

$$
\begin{gathered}
\dot{\xi}=1+z \gamma_{1}(\xi, v, z), \quad \dot{v}=z \gamma_{2}(\xi, v, z), \\
\varepsilon \dot{z}=\varkappa(\xi, v) z+z^{2}+\varepsilon \Delta(\xi, v, z),
\end{gathered}
$$

где $\gamma_{1}, \gamma_{2}$ - некоторые достаточно гладкие функции, а функции $\varkappa(\xi, v)$ и $\Delta(\xi, v, z)$ получаются соответственно из

$$
\varphi(x)-\psi(x), \quad-(\nabla \varphi(x), f(x, z+\varphi(x)))
$$

при указанной замене координат. Отметим, далее, что поскольку система (16) рассматривается в некоторой достаточно малой окрестности начала координат, то в силу первого равенства (16) переменную $\xi$ в ней можно взять за новое время. В итоге получим систему

$$
\frac{\varepsilon d z}{d \xi}=\varkappa(\xi, v) z+H(\xi, v, z)+\varepsilon \Delta_{1}(\xi, v, z), \quad \frac{d v}{d \xi}=z \Delta_{2}(\xi, v, z),
$$

где гладкая функция $H$ такова, что

$$
H(\xi, v, 0) \equiv H_{z}^{\prime}(\xi, v, 0) \equiv 0
$$

a $\Delta_{1}$ и $\Delta_{2}$ задаются равенствами

$$
\Delta_{1}=\frac{\Delta(\xi, v, z)}{1+z \gamma_{1}(\xi, v, z)}, \quad \Delta_{2}=\frac{\gamma_{2}(\xi, v, z)}{1+z \gamma_{1}(\xi, v, z)}
$$

Перед вьполнением третьего шага нормализации вспомним, что при сведении задачи к локальной мы рассматривали гладкие поверхности (ленты) $y=S_{j}(x, \varepsilon), j=1,2$, образованные траекториями системы (1). В исследуемой нами окрестности точки $\left(x_{0}\right.$, $\left.\varphi\left(x_{0}\right)\right)$ эти поверхности находятся на расстоянии порядка $\varepsilon$ от поверхности $\Gamma_{1}$, т.е. в новых координатах от поверхности $z=0$. Поэтому целесообразно предварительно сделать в системе (17) замену $z / \varepsilon \rightarrow z$, в результате которой с учетом свойств (18) функции $H$ она преобразуется к виду

$$
\frac{\varepsilon d z}{d \xi}=\varkappa(\xi, v) z+\Delta_{3}(\xi, v)+\varepsilon \Delta_{4}(\xi, v, z, \varepsilon), \quad \frac{d v}{d \xi}=\varepsilon \Delta_{5}(\xi, v, z, \varepsilon)
$$

Здесь $\Delta_{j}, j=3,4,5,-$ некоторые достаточно гладкие по совокупности переменных функции, причем в силу (19)

$$
\Delta_{3}(\xi, v)=\Delta(\xi, v, 0) .
$$


Далее заметим, что в силу условий 1,2

$$
\begin{gathered}
\varkappa(0, v)=\left.(\varphi(x)-\psi(x))\right|_{x \in l_{0}} \equiv 0, \\
\varkappa_{\xi}^{\prime}(0, v)=\left.(\nabla \varphi(x)-\nabla \psi(x), f(x, \varphi(x)))\right|_{x \in l_{0}}>0 .
\end{gathered}
$$

Следовательно, можно сделать гладкую замену, при которой имело бы место равенство

$$
\frac{d \tilde{\xi}}{d \xi}=\frac{\varkappa(\xi, v)}{\tilde{\xi}}
$$

(переменная $v$ рассматривается здесь как параметр). Действительно, решая это дифференциальное уравнение, получим гладкое решение

$$
\tilde{\xi}=\xi \sqrt{\frac{2}{\xi^{2}} \int_{0}^{\xi} \varkappa(s, v) d s} .
$$

Итак, на третьем, последнем шаге нормализации выполним в системе (20) замену (22), а затем переменную $\tilde{\xi}$ снова обозначим через $\xi$. В итоге получаем искомую нормальную форму системы (1), которая имеет вид

$$
\frac{\varepsilon d z}{d \xi}=\xi z+\Omega(\xi, v)+\varepsilon \Theta_{1}(\xi, v, z, \varepsilon), \quad \frac{d v}{d \xi}=\varepsilon \Theta_{2}(\xi, v, z, \varepsilon) .
$$

Здесь все функции достаточно гладко зависят от своих переменных, причем в силу (19), (21) справедливо равенство

$$
\Omega(0, v)=-\frac{\Phi\left(v+s_{0}\right)}{\left.\sqrt{\varkappa_{\xi}^{\prime}(0, v)} \omega(x, \varphi(x))\right|_{x=\gamma\left(s_{0}+v\right)}},
$$

где $\Phi(s)$ - функция (7). А отсюда и из условия 4 вытекает, что

$$
\Omega(0,0)=0, \quad \Omega_{v}^{\prime}(0,0) \neq 0 .
$$

На заключительном этапе доказательства теоремы фиксируем достаточно малое число $q>0$ и при $-q \leqslant \xi \leqslant q$ рассмотрим для системы (23) краевую задачу с граничными условиями

$$
\left.z\right|_{\xi=-q}=\left.\Sigma_{1}(\xi, v, \varepsilon)\right|_{\xi=-q},\left.\quad z\right|_{\xi=q}=\left.\Sigma_{2}(\xi, v, \varepsilon)\right|_{\xi=q}
$$

где гладкие по совокупности переменных функции

$$
\Sigma_{j},\left.\quad \Sigma_{j}\right|_{\varepsilon=0}=-\frac{\Omega(\xi, v)}{\xi}, \quad j=1,2,
$$

определенные в некоторых достаточно малых окрестностях точек $\xi=-q, v=0$ и $\xi=q$, $v=0$ соответственно, представляют собой введенные вьше интегральные многообразия (ленты) $y=S_{j}(x, \varepsilon), j=1,2$, в новых переменных.

В силу равенств (15) нас интересует решение краевой задачи (23), (25) (если таковое существует) с нулевым приближением

$$
v=0, \quad z=z_{0}(\xi) \equiv-\frac{\Omega(\xi, 0)}{\xi},
$$

где, подчеркнем, $z_{0}(\xi) \in C^{\infty}[-q, q]$ (см. первое свойство (24)). 
Лемма. При всех достаточно мальх $\varepsilon>0$ краевая задача (23), (25) имеет единственное решение $v=v(\xi, \varepsilon), z=z(\xi, \varepsilon)$ с нулевым приближением (26), для которого равномерно по $-q \leqslant \xi \leqslant q$ справедливы асимптотические представления

$$
v=\sum_{k=1}^{\infty} \varepsilon^{k} v_{k}(\xi), \quad z=z_{0}(\xi)+\sum_{k=1}^{\infty} \varepsilon^{k} z_{k}(\xi)
$$

ДокАЗАТЕЛЬСТво. Изложим сначала алгоритм нахождения коэффициентов рядов (27). С этой целью подставим указанные ряды в систему (23) и будем последовательно приравнивать коэффициенты при одинаковых степенях $\varepsilon$.

На первом шаге алгоритма приходим к системе

$$
\frac{d v_{1}}{d \xi}=\Theta_{2}\left(\xi, 0, z_{0}(\xi), 0\right), \quad \xi z_{1}=z_{0}^{\prime}(\xi)-\Omega_{v}^{\prime}(\xi, 0) v_{1}-\Theta_{1}\left(\xi, 0, z_{0}(\xi), 0\right),
$$

анализ которой достаточно прост. Действительно, из первого уравнения (28) функция $v_{1}(\xi)$ определяется с точностью до константы, которую мы выберем так, чтобы функция $z_{1}(\xi)$ была гладкой в окрестности точки $\xi=0$. А именно положим

$$
v_{1}(0)=\frac{\left(z_{0}^{\prime}(0)-\Theta_{1}\left(0,0, z_{0}(0), 0\right)\right)}{\Omega_{v}^{\prime}(0,0)},
$$

что возможно в силу второго соотношения (24).

На шаге с номером $k \geqslant 2$ имеем аналогичную (28) систему

$$
\frac{d v_{k}}{d \xi}=R_{k}(\xi), \quad \xi z_{k}=G_{k}(\xi)-\Omega_{v}^{\prime}(\xi, 0) v_{k}
$$

где функции $R_{k}, G_{k} \in C^{\infty}[-q, q]$ зависят только от $v_{m}(\xi)$ и $z_{m}(\xi)$ с номерами $m \leqslant k-1$. Решая затем уравнение для $v_{k}$ и выбирая начальное условие $v_{k}(0)=G_{k}(0) / \Omega_{v}^{\prime}(0,0)$, получим функцию $z_{k}(\xi)$, гладкую в окрестности точки $\xi=0$, и т.д.

Для обоснования приведенного алгоритма нам потребуются два специальных семейства решений системы (23), каждое из которых удовлетворяет одному из граничных условий (25). Указанные семейства определяются следуюшим образом. Фиксируем произвольное натуральное $k_{0} \geqslant 2$ и подставим в $(23)$ выражения

$$
v=v_{k_{0}}^{j}(\xi, \varepsilon)+h_{j}, \quad z=z_{k_{0}}(\xi, \varepsilon)+g_{j}, \quad j=1,2,
$$

где

$$
v_{k_{0}}^{j}(\xi, \varepsilon)=\sum_{k=1}^{k_{0}-1} \varepsilon^{k} v_{k}(\xi)+\varepsilon^{k_{0}} \delta_{j}, \quad z_{k_{0}}(\xi, \varepsilon)=z_{0}(\xi)+\sum_{k=1}^{k_{0}-1} \varepsilon^{k} z_{k}(\xi)
$$

a $\delta_{j}, j=1,2,-$ пока произвольные (подлежащие определению в дальнейшем) постоянные. В итоге для $h_{j}=h_{j}\left(\xi, \varepsilon, \delta_{j}\right), g_{j}=g_{j}\left(\xi, \varepsilon, \delta_{j}\right), j=1,2$, получим некоторые системы дифференциальных уравнений, которые дополним начальными условиями

$$
\left.h_{j}\right|_{\xi=(-1)^{j} q}=0,\left.\quad g_{j}\right|_{\xi=(-1)^{j} q}=\left.\left(\Sigma_{j}\left(\xi, v_{k_{0}}^{j}(\xi, \varepsilon), \varepsilon\right)-z_{k_{0}}(\xi, \varepsilon)\right)\right|_{\xi=(-1)^{j} q} .
$$


При $j=1$ описанная последовательность действий приводит для определения функций $h_{1}\left(\xi, \varepsilon, \delta_{1}\right), g_{1}\left(\xi, \varepsilon, \delta_{1}\right)$ к системе

$$
\begin{aligned}
\frac{\varepsilon d g_{1}}{d \xi} & =a\left(\xi, \varepsilon, \delta_{1}\right) g_{1}+b\left(\xi, \varepsilon, \delta_{1}\right) h_{1}+C\left(g_{1}, h_{1}, \xi, \varepsilon, \delta_{1}\right)+\varepsilon^{k_{0}} u_{*}\left(\xi, \varepsilon, \delta_{1}\right), \\
\frac{d h_{1}}{d \xi} & =\varepsilon D\left(g_{1}, h_{1}, \xi, \varepsilon, \delta_{1}\right)+\varepsilon^{k_{0}} u_{* *}\left(\xi, \varepsilon, \delta_{1}\right)
\end{aligned}
$$

в которой все коэффициенты гладко зависят от своих переменных, причем

$$
\begin{gathered}
a\left(\xi, \varepsilon, \delta_{1}\right)=\xi+O(\varepsilon), \quad b\left(\xi, \varepsilon, \delta_{1}\right)=\Omega_{v}^{\prime}(\xi, 0)+O(\varepsilon), \\
C\left(0,0, \xi, \varepsilon, \delta_{1}\right) \equiv C_{g_{1}}^{\prime}\left(0,0, \xi, \varepsilon, \delta_{1}\right) \equiv C_{h_{1}}^{\prime}\left(0,0, \xi, \varepsilon, \delta_{1}\right) \equiv D\left(0,0, \xi, \varepsilon, \delta_{1}\right) \equiv 0 .
\end{gathered}
$$

Дополним затем получившуюся систему начальными условиями (30) при $j=1$ и перейдем от нее к системе интегральных уравнений

$$
\begin{aligned}
g_{1}= & g_{1}\left(-q, \varepsilon, \delta_{1}\right) \exp \left\{\frac{1}{\varepsilon} \int_{-q}^{\xi} a\left(s, \varepsilon, \delta_{1}\right) d s\right\} \\
& +\frac{1}{\varepsilon} \int_{-q}^{\xi} \exp \left\{\frac{1}{\varepsilon} \int_{\tau}^{\xi} a\left(s, \varepsilon, \delta_{1}\right) d s\right\}\left[b ( \tau , \varepsilon , \delta _ { 1 } ) \left(\varepsilon \int_{-q}^{\tau} D\left(g_{1}, h_{1}, \sigma, \varepsilon, \delta_{1}\right) d \sigma\right.\right. \\
& \left.\left.+\varepsilon^{k_{0}} \int_{-q}^{\tau} u_{* *}\left(\sigma, \varepsilon, \delta_{1}\right) d \sigma\right)+C\left(g_{1}, h_{1}, \tau, \varepsilon, \delta_{1}\right)+\varepsilon^{k_{0}} u_{*}\left(\tau, \varepsilon, \delta_{1}\right)\right] d \tau \\
h_{1}= & \varepsilon \int_{-q}^{\xi} D\left(g_{1}, h_{1}, s, \varepsilon, \delta_{1}\right) d s+\varepsilon^{k_{0}} \int_{-q}^{\xi} u_{* *}\left(s, \varepsilon, \delta_{1}\right) d s .
\end{aligned}
$$

Исследование системы (32), (33) опирается на асимптотическое равенство

$$
g_{1}\left(-q, \varepsilon, \delta_{1}\right)=O\left(\varepsilon^{k_{0}}\right)
$$

справедливое в силу описанного вьше способа нахождения коэффициентов рядов (27), и легко проверяемую оценку

$$
\max _{-q \leqslant \xi \leqslant 0} \frac{1}{\varepsilon} \int_{-q}^{\xi} \exp \left\{\frac{1}{\varepsilon} \int_{\tau}^{\xi} a\left(s, \varepsilon, \delta_{1}\right) d s\right\} d \tau \leqslant \frac{M}{\sqrt{\varepsilon}}, \quad M=\text { const }>0 .
$$

Используя эти факты вместе со свойствами (31), убеждаемся, что оператор, порожденный правыми частями уравнений (32), (33) в пространстве

$$
\left(g_{1}, h_{1}\right) \in C[-q, 0] \times C[-q, 0]
$$

преобразует в себя множество $B_{1} \times B_{2}$, где $B_{j}, j=1,2,-$ некоторые шары в $C[-q, 0]$ с центрами в нуле и радиусами порядка $\varepsilon^{k_{0}-1 / 2}$ и $\varepsilon^{k_{0}}$ соответственно, и является сжимающим. Поэтому из $(32),(33)$ единственным образом определяются гладкие по совокупности переменных $\xi, \delta_{1}$ функции $g_{1}\left(\xi, \varepsilon, \delta_{1}\right), h_{1}\left(\xi, \varepsilon, \delta_{1}\right)$ :

$$
\max _{-q \leqslant \xi \leqslant 0}\left(\left|g_{1}\right|+\left|\frac{\partial g_{1}}{\partial \delta_{1}}\right|\right) \leqslant M_{1} \varepsilon^{k_{0}-1 / 2}, \quad \max _{-q \leqslant \xi \leqslant 0}\left(\left|h_{1}\right|+\left|\frac{\partial h_{1}}{\partial \delta_{1}}\right|\right) \leqslant M_{2} \varepsilon^{k_{0}},
$$


где $M_{j}=$ const $>0, j=1,2$. Аналогичным образом определяем на отрезке $0 \leqslant \xi \leqslant q$ и фигурирующие в $(29)$ функции $g_{2}\left(\xi, \varepsilon, \delta_{2}\right), h_{2}\left(\xi, \varepsilon, \delta_{2}\right)$.

Проделанные построения сводят проблему нахождения требуемого решения краевой задачи $(23),(25)$ к отысканию двух имеющихся в запасе параметров $\delta_{1}, \delta_{2}$ из условий “сшивания" решений $(29)$ при $\xi=0$, т.е. из равенств

$$
\varepsilon^{k_{0}} \delta_{1}+\left.h_{1}\right|_{\xi=0}=\varepsilon^{k_{0}} \delta_{2}+\left.h_{2}\right|_{\xi=0},\left.\quad g_{1}\right|_{\xi=0}=\left.g_{2}\right|_{\xi=0}
$$

Проанализируем сначала первое из уравнений системы (35). С этой целью заметим, что $u_{* *}\left(\xi, 0, \delta_{1}\right)=u_{* *}^{0}(\xi)$, где $u_{* *}^{0}(\xi)$ - некоторая гладкая, не зависящая от $\delta_{1}$ функция. Учитьвая данное обстоятельство вместе с оценками (34) в уравнении (33), заключаем, что равномерно по $\xi \in[-q, 0]$

$$
h_{1}=\varepsilon^{k_{0}} \int_{-q}^{\xi} u_{* *}^{0}(s) d s+O\left(\varepsilon^{k_{0}+1 / 2}\right), \quad \frac{\partial h_{1}}{\partial \delta_{1}}=O\left(\varepsilon^{k_{0}+1 / 2}\right) .
$$

Объединяя, далее, эти равенства с аналогичными представлениями

$$
h_{2}=-\varepsilon^{k_{0}} \int_{\xi}^{q} u_{* *}^{0}(s) d s+O\left(\varepsilon^{k_{0}+1 / 2}\right), \quad \frac{\partial h_{2}}{\partial \delta_{2}}=O\left(\varepsilon^{k_{0}+1 / 2}\right),
$$

заключаем, что из первого уравнения $(35) \delta_{2}$ выражается через $\delta_{1}$ :

$$
\delta_{2}=\delta_{1}+\int_{-q}^{q} u_{* *}^{0}(s) d s+O(\sqrt{\varepsilon})
$$

Для исследования второго уравнения (35) учтем более детально структуру функции $u_{*}$ :

$$
u_{*}=\delta_{1} \Omega_{v}^{\prime}(\xi, 0)+u_{*}^{0}(\xi)+O(\varepsilon) \text {, }
$$

где $u_{*}^{0}(\xi)$ - некоторая гладкая функция (ее явное выражение не потребуется). После этого сделаем в интегралах, стоящих в правой части $(32)$, замены $s / \sqrt{\varepsilon} \rightarrow s, \tau / \sqrt{\varepsilon} \rightarrow \tau$, а затем воспользуемся свойствами $(31),(34),(37)$. В результате придем к асимптотическим равенствам

$$
g_{1}\left(0, \varepsilon, \delta_{1}\right)=\varepsilon^{k_{0}-1 / 2}\left(\varkappa_{1,1} \delta_{1}+\varkappa_{1,2}\right)+O\left(\varepsilon^{k_{0}}\right), \quad \frac{\partial g_{1}}{\partial \delta_{1}}\left(0, \varepsilon, \delta_{1}\right)=\varepsilon^{k_{0}-1 / 2} \varkappa_{1,1}+O\left(\varepsilon^{k_{0}}\right)
$$

где значение постоянной $\varkappa_{1,2}$ несущественно, а

$$
\varkappa_{1,1}=\Omega_{v}^{\prime}(0,0) \int_{-\infty}^{0} \exp \left(-\frac{\tau^{2}}{2}\right) d \tau \neq 0
$$

(см. (24)). Объединяя их с аналогичными равенствами для $g_{2}$ :

$$
g_{2}\left(0, \varepsilon, \delta_{2}\right)=\varepsilon^{k_{0}-1 / 2}\left(\varkappa_{2,1} \delta_{2}+\varkappa_{2,2}\right)+O\left(\varepsilon^{k_{0}}\right), \quad \frac{\partial g_{2}}{\partial \delta_{2}}\left(0, \varepsilon, \delta_{2}\right)=\varepsilon^{k_{0}-1 / 2} \varkappa_{2,1}+O\left(\varepsilon^{k_{0}}\right)
$$


где

$$
\varkappa_{2,1}=-\Omega_{v}^{\prime}(0,0) \int_{0}^{\infty} \exp \left(-\frac{\tau^{2}}{2}\right) d \tau \neq 0
$$

приходим к выводу, что второе уравнение (35) преобразуется к виду

$$
F\left(\delta_{1}, \delta_{2}, \varepsilon\right)=0
$$

где

$$
\begin{gathered}
F=\Omega_{v}^{\prime}(0,0) \sqrt{\frac{\pi}{2}}\left(\delta_{1}+\delta_{2}\right)+\varkappa_{1,2}-\varkappa_{2,2}+O(\sqrt{\varepsilon}), \\
\frac{\partial F}{\partial \delta_{j}}=\Omega_{v}^{\prime}(0,0) \sqrt{\frac{\pi}{2}}+O(\sqrt{\varepsilon}), \quad j=1,2 .
\end{gathered}
$$

И наконец, подставляя в (38) равенство (36) и применяя к получившемуся уравнению теорему о неявной функции, однозначно определяем ограниченные функции $\delta_{1}=\delta_{1}(\varepsilon)$, $\delta_{2}=\delta_{2}(\varepsilon)$. Лемма доказана.

Отметим, что аналог установленной леммы содержится в работе [5], где система вида (17) рассматривается как самостоятельньй объект исследования.

Для завершения обоснования теоремы остается совершить обратный переход от локальной постановки задачи к глобальной, а точнее говоря, продолжить доставляемую леммой траекторию системы (23) по лентам $y=S_{1}(x, \varepsilon)$ и $y=S_{2}(x, \varepsilon)$ до пересечения с кривыми $l^{-}$и $l^{+}$соответственно. В результате получим единственную траекторию системы (1) с концами на $l^{-}$и $l^{+}$и с требуемьм нулевым приближением $L$. Теорема доказана.

Сделаем еще два небольших замечания. Во-первых, отметим, что все вышеизложенное очевидным образом переносится на случай, когда правые части системы (1) - функции $f$ и $g$ - зависят дополнительно и от параметра $\varepsilon$. Во-вторых, укажем, что в рассматриваемой задаче (1) фазовые траектории, не являющиеся траекториями-утками, исследовались в статье [6].

3. Заключение. Следует подчеркнуть, что используемая выше стратегия “охоты на уток" отличается от предложенной в статье [2]. Действительно, в [2] предполагалось, что правые части рассматриваемой системы зависят от некоторого вспомогательного параметра, необходимого в случае одной медленной переменной для склейки траеторий-уток в окрестности точки пересечения кривых медленных движений. В нашей же ситуации указанная склейка осуществляется за счет варьирования начальных условий на кривых $l^{-}, l^{+}$или, что то же самое, на лентах $y=S_{j}(x, \varepsilon), j=1,2$. Более того, при количестве медленных переменных, равном $n \geqslant 3$, появление новых степеней свободы приводит к эффекту неединственности траектории-утки с концами на заданных $(n-1)$-мерных поверхностях $l^{-}, l^{+}$и с фиксированньм нулевым приближением $L$. Точнее говоря, опираясь на развитую выше технику, можно показать, что при вьполнении аналогов условий $1-4$ в этом случае имеется целое $(n-2)$-параметрическое семейство таких траекторий. 
Остановимся еще на одной стратегии “охоты на уток", которую уместнее назвать стратегией “выращивания уток”. С этой целью рассмотрим аналогичную (1) систему

$$
\dot{x}=f(x, y), \quad \varepsilon \dot{y}=g(x, y, \mu),
$$

правая часть $g$ которой гладко зависит от некоторого вспомогательного параметра $\mu$. Будем считать, что при $\mu=0$ справедливы условия $1-3$, а вместо условия 4 вьполняется неравенство $g_{\mu}^{\prime}(x, y, 0) \neq 0$ в точках кривой $l$. Тогда, используя те же конструкции, что и вьше, можно показать (см. также [5]) существование такого значения

$$
\mu=\mu_{*}(\varepsilon), \quad \mu_{*}(\varepsilon)=\frac{\varepsilon \Phi\left(s_{0}\right)}{g_{\mu}^{\prime}\left(x_{0}, \varphi\left(x_{0}\right), 0\right)}+O\left(\varepsilon^{2}\right)
$$

что при $\mu=\mu_{*}(\varepsilon)$ система (39) имеет траекторию-утку с наперед заданным нулевым приближением $L$ (см. рис. 3). Таким образом, при подходящем выборе параметра $\mu$ можно добиться выживания при $\varepsilon>0$ любой из траекторий семейства (6). Добавим, наконец, что если считать параметр $\mu$ гладкой функцией переменных $x$ и $\varepsilon$, то при некотором $\mu=\mu_{*}(x, \varepsilon), \mu_{*}(x, 0) \equiv 0$, можно обеспечить выживание всех траекторий-уток из указанного семейства. (См. статью [7], где эта идея реализована в близкой ситуации.)

В заключение отметим, что при отсутствии вспомогательного параметра $\mu$ равенство $\Phi\left(s_{0}\right)=0$, достаточное для существования у системы (1) траектории-утки с выбранным нулевым приближением, является в то же время и необходимым. В самом деле, как следует из содержащихся в [2], [6] построений, при его нарушении траектория системы (1) с начальньпи условиями $\left.(x, y)\right|_{t=0}=\left(x^{-}, \varphi\left(x^{-}\right)\right)$имеет нулевое приближение одного из двух типов, показанньх на рис. 5 .

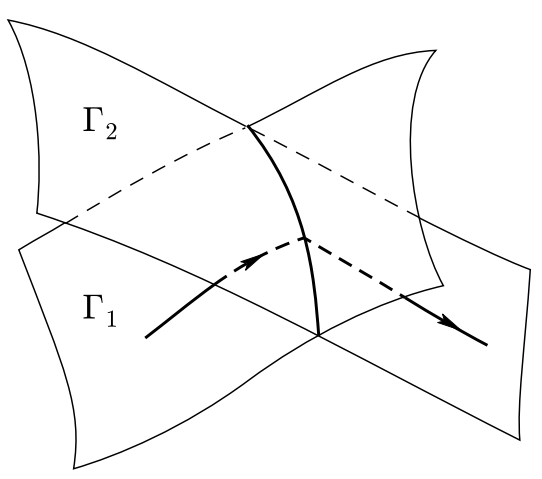

a)

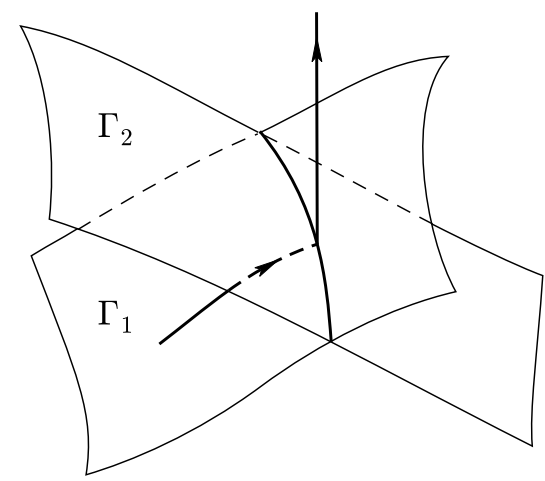

б)

Рис. 5

Отметим еще, что в случае а), реализующемся при $\Phi\left(s_{0}\right)>0$, интересующая нас траектория переходит с участка $\Gamma_{1}^{-}$на устойчивьй участок поверхности $\Gamma_{2}$, см. рис. 5 a). В случае же $\Phi\left(s_{0}\right)<0$, показанном на рис. 5 б), в точке $\left(x_{0}, \varphi\left(x_{0}\right)\right)$ происходит ее "срыв" с поверхности $\Gamma_{1}$ и последующее асимптотически быстрое движение вдоль вертикальной прямой $x=x_{0}$. 
Итак, при некоторой общности положения в системе (1) “выживают" лишь траектории-утки с отдельно взятыми изолированньми нулевыми приближениями (6), на которых обращается в нуль функция (7). Эти траектории служат своего рода сепаратрисами, разделяющими массивы траекторий системы (1) с различным асимптотическим поведением (см. рис. 5).

Авторы считают своим прямым долгом выразить искреннюю признательность рецензенту за его глубокое внимание к настоящей работе и исключительно полезные конструктивные замечания, несомненно способствовавшие улучшению ее содержания.

\section{СПИСОК ЦИТИРОВАННОЙ ЛИТЕРАТУРЫ}

[1] Мищенко Е.Ф., Колесов Ю. С., Колесов А. Ю., Розов Н. Х. Периодические движения и бифуркационные процессы в сингулярно возмущенных системах. М.: Физматлит, 1995.

[2] Колесов А. Ю., Мищенко Е.Ф., Розов Н. Х. Решение сингулярно возмущенных краевых задач методом "охоты на уток" // Тр. МИАН. 1999. Т. 224. С. 187-207.

[3] Мищенко Е.Ф., Розов Н. Х. Дифференциальные уравнения с малым параметром и релаксационные колебания. М.: Наука, 1975.

[4] Васильева А. Б., Бутузов В. Ф. Асимптотические разложения решений сингулярно возмущенных уравнений. М.: Наука, 1973.

[5] Соболев В.А., Шепакина Е. А. Траектории-утки в одной задаче теории горения // Дифференц. уравнения. 1996. Т. 32. №9. С. 1175-1184.

[6] Lebovitz N. R., Schaar R. J. Exchange of stabilities in autonomous systems // Stud. Appl. Math. 1975. V. 54. P. 229-260.

[7] Соболев В.А., Шепакина Е. А. Интегральные поверхности со сменой устойчивости и траектории-утки // Изв. РАЕН. Сер. МММИУ. 1997. Т. 1. № 3. С. 176-187.

(А. С. Бобкова, Н. Х. Розов) Московский государственный

Поступило университет им. М.В. Ломоносова

22.02 .2001

(А. Ю. Колесов) Ярославский государственный университет им. П. Г. Демидова

Исправленный вариант

05.11.2001 